\title{
Case Report: Therapeutic Threshold for Rifampicin-Resistant Tuberculosis in a Patient from Maputo, Mozambique
}

\author{
Lorena D. M. Gonzaga, ${ }^{1}$ Tinne Gils, ${ }^{2 \star}$ Tom Decroo, ${ }^{2,3}$ Bart K. M. Jacobs, ${ }^{2}$ and Lutgarde Lynen ${ }^{2}$ \\ ${ }^{1}$ Centro de Saude Primeiro de Maio, Maputo, Mozambique; ${ }^{2}$ Department of Clinical Sciences, Institute of Tropical Medicine, Antwerp, Belgium; \\ ${ }^{3}$ Research Foundation Flanders, Brussels, Belgium
}

\begin{abstract}
We present a case of a patient in Mozambique, who initiated treatment for rifampicin-resistant tuberculosis (RR-TB) without proof of resistance. For this patient, we estimated the probability of RR-TB using likelihood ratios of clinical arguments. The probability of RR-TB in Mozambique, positive HIV status, and treatment failure after a first treatment and after retreatment were included as confirming arguments, and a rapid molecular test showing rifampicin susceptibility as excluding argument. The therapeutic threshold to start treatment for RR-TB is unknown, but probably lower than $47 \%$ and should be calculated to guide clinical decisions.
\end{abstract}

\section{INTRODUCTION}

Rollout of rapid molecular tests to detect rifampicin (RIF) resistance $(\mathrm{RR})$ has likely led to a decrease in empirical treatment for RIF-resistant tuberculosis (RR-TB). ${ }^{1}$ Most RRconferring mutations are situated in the rifampicin resistance determining region (RRDR), targeted by rapid molecular tests ${ }^{2,3}$ such as Xpert ${ }^{\circledR}$ Mycobacterium tuberculosis (MTB)/ RIF and Xpert ${ }^{\circledR}$ MTB/RIF Ultra (Xpert MTB/RIF, Cepheid, Sunnyvale, CA) and line-probe assays (LPAs; GenoType ${ }^{\circledR}$ MTBDRplus and GenoType ${ }^{\circledR}$ MTBDRsl, HAIN Lifescience, Nehren, Germany). ${ }^{4,5}$ Both tests report on the detection of MTB and of RR. When diagnosis of RR-TB depends on such tests, RR-TB patients with mutations outside of the RRDR may be repeatedly treated with first-line regimens, mostly without success. $^{4,6}$

The probability of disease required to treat a patient, or the therapeutic threshold, with equipoise between treating and not treating, ${ }^{7}$ has not yet been estimated for RR-TB. ${ }^{8}$ Current guidelines recommend all TB patients should be tested for RR. ${ }^{9}$ The sensitivity and specificity of Xpert MTB/RIF to detect $\mathrm{RR}$ are $96 \%$ and $98 \%$, respectively. ${ }^{10}$ In case of Xpert MTB+/ RIF- (MTB detected, RR not detected), first-line treatment is recommended. ${ }^{9}$ In patients with a very high probability of RR$\mathrm{TB}$, treatment can be started regardless of test results, based on clinical decision-making. We determined this probability for a Mozambican patient when clinicians started RR-TB treatment without bacteriological proof of RR.

Mozambique is a high TB- and RR-TB-burden country. An estimated $3.7 \%$ of new, $20 \%$ of previously treated patients, and $80 \%$ of patients with repetitive first-line treatment failure have RR-TB. ${ }^{11}$ Drug susceptibility testing (DST) by Xpert MTB/ RIF is recommended for all TB patients. Health facilities without Xpert MTB/RIF send sputum samples to a nearby facility and manage TB patients based on smear microscopy while waiting for results. In the capital Maputo, DST beyond RIF is carried out at the Central Hospital for retreatment, treatment failure, or suspected RR-TB cases.

Likelihood ratios (LRs) were calculated for clinical arguments and Xpert MTB/RIF results for one patient, using probabilities and odds ratios from the literature. The confirming

*Address correspondence to Tinne Gils, Department of Clinical Sciences, Institute of Tropical Medicine, Nationalestraat 155, Antwerp 2000, Belgium. E-mail: tgils@itg.be power is the positive LR or the number of times a positive test result is more likely in a diseased versus a non-diseased person. The excluding power is the inverse of a negative LR or the number of times more likely a negative test result is in a non-diseased versus a diseased person. Excluding and confirming powers are not directly influenced by disease prevalence. $^{12}$

$$
\begin{aligned}
& \text { excluding power }=\frac{\text { specificity }}{1-\text { sensitivity }} \\
& \text { confirming power }=\frac{\text { sensitivity }}{1-\text { specificity }} .
\end{aligned}
$$

The estimated probability of RR-TB in Mozambique was converted to odds and multiplied by the LRs of confirming arguments before testing. That result was multiplied with the excluding power of Xpert MTB+/RIF-. After accounting for all arguments, the probability of RR-TB and its variation were estimated. Uncertainty intervals (Uls) were constructed for log-odds, odds, and probabilities at each step by selecting the relevant quantiles from 1,000 independent estimates calculated based on random draws from the relevant power and probability distributions. $R$ version 3.5 .2 was used for analy$\operatorname{sis}^{13}$ (Supplemental File 1: detailed methodology).

In a clinical setting, the power of arguments can be estimated based on clinical expertise (e.g., strong) or rounded up or down to obtain integer numbers and then categorized. Such intuitive approximation of an LR can be converted to a logodds scale (Table 1). ${ }^{14}$ The patient was informed about the purpose of this study and signed consent.

\section{CASE STUDY}

The patient was a 40-year-old woman from rural southern Mozambique. In 2012, she was diagnosed with HIV and started first-line antiretroviral therapy (lamivudine, tenofovir, and efavirenz). She reported having a TB diagnosis and TB treatment at least twice: in 2014 for 6 months (RIF, isoniazid, ethambutol, pyrazinamide) and later for 8 months with the same drugs strengthened with 2 months of streptomycin. She interrupted the last regimen as her clinical presentation worsened. We considered at least two episodes of treatment failure.

In May 2018, the patient presented with productive cough, thoracic pain, wasting, no fever, normal blood pressure, a respiratory rate of 23 counts per minute, wheezing, and a 
TABLE 1

Effect of confirming and excluding power on probability of disease on the log-odds scale

\begin{tabular}{llc}
\hline Rounded power & Strength & Steps on the log-odds scale \\
\hline $60-200$ & Very strong & 2 \\
$20-50$ & Strong & 1.5 \\
$6-15$ & Good & 1 \\
$2-5$ & Weak & 0.5 \\
1 & Useless & 0 \\
\hline${ }^{*}$ Confirming or excluding power can range between 0 and infinity. The power is rarely 200 or \\
more, whereas power lower than 1 means the test is useless or should have its outcomes \\
reversed. \\
† If confirming power, add the respective number of steps, if excluding power, subtract \\
steps (unit in log ${ }_{10}$ odds).
\end{tabular}

positive sputum smear microscopy with high bacillary load. She had no known RR-TB contacts. Despite Xpert MTB/RIF showing MTB+/RIF-, an RR-TB treatment containing levofloxacin, capreomycin, ethionamide, cycloserine, pyrazinamide, and ethambutol was started. In June 2018, LPA DST showed $\mathrm{RR}$ to levofloxacin, ethionamide, and isoniazid, and confirmed RIF susceptibility. Treatment was modified to contain bedaquiline, delamanid, clofazimine, linezolid, and para-aminosalicylic acid. The patient had smear conversion and negative cultures from month 5 . The detailed clinical history and chest X-ray are available as Supplemental Files 2 and 3.

After including all arguments, the estimated probability of RR-TB in this patient was 46.6\% (95\% UI: 25.0-72.0; Table 2, Figure 1). An alternative starting point was a pretest probability of RR-TB in retreatment cases in Mozambique of $20 \%$ (95\% Cl: $5.2-40)$. When including the probability of HIV of $36 \%$, assuming the same HIV prevalence in new and retreatment TB cases, the probability of RR-TB increases to 24.0\% (95\% UI: 8.1-54.2) among HIV-positive patients with a TB history. ${ }^{11,15}$

\section{DISCUSSION}

We estimated the probability of RR-TB in an HIV-positive TB patient with retreatment failure and a susceptible RIF-DST result in Mozambique at 46.6\% (95\% UI: 25.0-72.0). At this probability, RR-TB treatment was started without delay. We illustrated the use of the log-odds scale to facilitate the process of clinical decision-making.

Because of its high sensitivity and specificity, ${ }^{10}$ Xpert $\mathrm{MTB}+/ \mathrm{RIF}+$ (MTB detected, RR detected), has a strong confirming power (approximately 50), whereas Xpert MTB+/RIFhas a lower, but still strong excluding power. However, RIFDST can miss RR-TB if mutations happened outside of the RRDR. $^{5,16,17}$ In Eswatini, 38/125 (30\%) of RR strains were not detected by Xpert MTB/RIF. ${ }^{3}$ In South Africa, 37/249 (15\%) samples identified as RS by Xpert MTB/RIF were reclassified as RR after sequencing. ${ }^{4}$ These patients could be wrongly treated for RS-TB, have worse treatment outcomes, and silently spread RR-TB. ${ }^{6}$ In Rwanda, when RIF-DST was not available or results delayed, $R R$ treatment initiation based on clinical decision-making reduced mortality. ${ }^{18}$ In patients with a high pretest probability of RR-TB, Xpert MTB+/RIF- is unlikely to lower the posttest probability below the therapeutic threshold, justifying empirical treatment. ${ }^{7}$

These scenarios, common in low-resource settings, show why establishment of a therapeutic threshold for RR-TB is important. The therapeutic threshold for pulmonary RS-TB in Rwanda was $2.6 \%$, rising to $12 \%$ when including regret factors such as treatment-related cost and morbidity. ${ }^{8,19}$ In our case, the therapeutic threshold is not equipoise between treating and not treating, but between treating for RS-TB or RR-TB. Compared with RS-TB, RR-TB treatment is longer, more toxic, and expensive, but disease-related mortality and morbidity is also higher. ${ }^{11}$ These regret factors should be considered when calculating the RR-TB threshold. ${ }^{20}$ Clinical

TABLE 2

Arguments used to estimate the probability of RR-TB in a patient in Mozambique

\begin{tabular}{|c|c|c|c|c|c|}
\hline \multirow[b]{3}{*}{1} & \multirow[b]{2}{*}{ Argument } & \multirow[b]{2}{*}{ Available data } & Odds of RR-TB & Probability of RR-TB & \multirow[b]{2}{*}{ Reference } \\
\hline & & & \multicolumn{2}{|c|}{ After accounting for the argument } & \\
\hline & $\begin{array}{l}\text { Newly diagnosed TB patient in } \\
\text { Mozambique }\end{array}$ & $\begin{array}{l}\text { Prevalence of RR-TB in Mozambique: } \\
3.7 \%(95 \% \mathrm{Cl}: 2.5-5.2)\end{array}$ & 0.038 & $3.7 \%$ (95\% Cl: $2.5-5.2)$ & 11 \\
\hline 2 & HIV positive & $\begin{array}{l}\text { OR of RR-TB in HIV-positive patients: } \\
1.49(95 \% \mathrm{Cl}: 0.73-3.06)\end{array}$ & 0.049 & 4.6\% (95\% UI: 2.5-8.7) & 24 \\
\hline \multirow[t]{2}{*}{3} & Treatment failure after a first treatment & $\begin{array}{l}\text { OR of initial RR-TB in retreatment cases } \\
\text { (after treatment failure of a first } \\
\text { treatment): } 7.24 \text { (95\% Cl: } 4.06-12.89)\end{array}$ & 0.246 & 19.8\% (95\% UI: 9.0-36.7) & 24 \\
\hline & & $\begin{array}{l}\text { Probability of acquiring RR-TB during a } \\
\text { first TB treatment (if no initial RR-TB) } \\
\text { that resulted in treatment failure: } 28.6 \% \\
(95 \% \mathrm{Cl}: 8.4-58.1)\end{array}$ & 0.745 & 42.7\% (95\% UI: 24.0-67.8) & 6 \\
\hline \multirow[t]{2}{*}{4} & Treatment failure after retreatment & $\begin{array}{l}\text { Likelihood ratio of retreatment failure in } \\
\text { patients with RR-TB acquired during } \\
\text { first treatment (before starting } \\
\text { retreatment): } 19.1 \text { (95\% Cl: 15.2-24.1) }\end{array}$ & 14.152 & 93.4\% (95\% UI: 85.4-97.5) & 6 \\
\hline & & $\begin{array}{l}\text { Probability of acquiring RR-TB during } \\
\text { retreatment if no initial RR-TB and } \\
\text { retreatment failure: } 31.9 \%(95 \% \mathrm{Cl} \text { : } \\
24.7-39.7) \text {. }\end{array}$ & 21.222 & $95.5 \%(95 \% \text { UI: } 90.4-98.3)^{\star}$ & 6 \\
\hline 5 & Xpert MTB+/RIF- & $\begin{array}{l}\text { Sensitivity: } 96 \% \text { (95\% Cl: } 94-97 \%) \text { and } \\
\text { specificity: } 98 \% \text { (95\% Cl: } 98-99) \text { of } \\
\text { Xpert to detect RR in adults }\end{array}$ & 0.873 & 46.6\% (95\% UI: 25.0-72.0)† & 10 \\
\hline
\end{tabular}



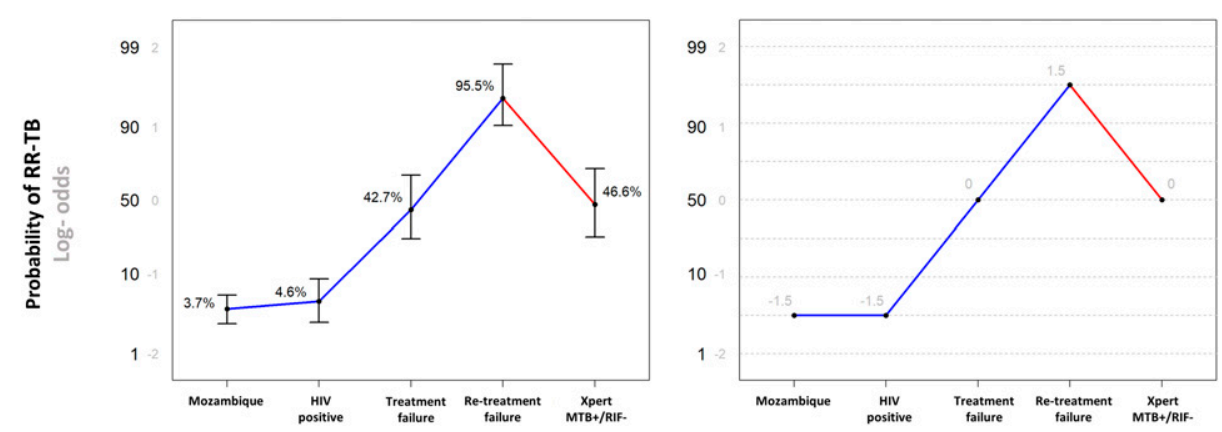

FIGURE 1. Estimated (left) and rounded (right) effect of confirming and excluding power on probability of RR-TB on a probability scale and a logodds scale in a patient in Mozambique. $Y$-axes are shown on a probability scale and a log-odds scale. Left: The impact of clinical arguments on the posttest probability of RR-TB with $95 \%$ uncertainty intervals. Prevalence of RR-TB in Mozambique (3.7\%) corresponds with a starting point on the log-odds scale of -1.42 . Confirming arguments move the probability of RR-TB up, and Xpert MTB+/RIF- lowers the probability of RR-TB. The estimated probability of RR-TB after testing was $47 \%$. Right: Rounded changes on the log-odds scale and the corresponding probabilities of RRTB. Very strong, strong, good, or weak confirming power allows to advance, respectively, 2, 1.5, 1, or 0.5 steps upward on the log-odds scale, and thus results in a higher posttest probability. The excluding power of an argument allows to regress downward and thus results in a lower posttest probability. The probability of having RR-TB in Mozambique reflects a starting point on the log-odds scale rounded to -1.5. HIV-positive is a "useless" argument, resulting in 0 steps on the log-odds scale. Treatment failure, retreatment failure are "strong" confirming and Xpert MTB+/RIF-a "strong" excluding arguments. The approximate result corresponds to a probability of $50 \%$. RIF = rifampicin; RR-TB = rifampicin-resistant tuberculosis; Xpert MTB+/RIF- = Xpert Mycobacterium tuberculosis detected/rifampicin resistance not detected. This figure appears in color at www.ajtmh.org.

vignettes, using varying clinical scenarios to create threshold curves, could assist. ${ }^{21,22}$

The large $\mathrm{Cl}$ in our estimation reflects uncertainty in the available clinical data, for example, on failure rates among RRTB patients and around accuracy estimates of the RIF-DST. As the estimated probability lied close to a one in two chance of RR-TB in this patient, the uncertainty was wider than when it would have been toward more extreme probabilities. This reflects dilemmas faced by clinicians daily.

In our estimations, most of the increased probability of RRTB after first treatment failure was attributed to the possibility of acquired RR during two unsuccessful first-line RIF-based treatments. RIF-DST with Xpert MTB/RIF could have a lower sensitivity after an unsuccessful outcome in a retreatment case, who could have tested false negative for RR-TB after their first unsuccessful TB treatment. If so, even without a first Xpert MTB/RIF result, the posttest probability of RR-TB would be higher. Because the excluding power is mainly determined by sensitivity, Xpert MTB+/RIF- would give a weaker argument against starting treatment. Our patient had a high bacillary load, in which case Xpert MTB/RIF can have a higher specificity for RIF-DST, ${ }^{23}$ but with minor impact on its excluding power.

We did not account for TB symptoms not specific to RRTB. HIV status was included because the patient was HIVpositive before her first TB episode, despite a nonsignificant weak association with RR-TB. ${ }^{24}$ We assumed conditional independence of arguments, although HIV positivity may be associated with a different chance of RR-TB after treatment failure than being HIV negative. Data from Bangladesh, used for calculation of RR probabilities after treatment and retreatment failure, are not necessarily applicable to Mozambique. ${ }^{6}$

The probability of RR-TB in HIV-positive retreatment cases estimated as alternative starting point (24\%) approached the estimate of the pretest probability of RR-TB in first treatment failure cases (20\%) because of initial RR-TB in HIV-positive patients, but was lower than the estimated $43 \%$ for a HIVpositive case with treatment failure, with a large UI. This could be explained by the fact that patients with a TB history include patients with treatment failure and those with reinfection. ${ }^{11}$

The therapeutic threshold is yet unknown for RR-TB, but probably less than $47 \%$. Establishing this threshold can guide clinical decision-making. ${ }^{8}$ Attributing confirming and excluding power to clinical arguments on a log-odds scale can help to rationalize the process.

Received August 5, 2020. Accepted for publication December 10, 2020.

Published online February 8, 2021.

Note: Supplemental files appear at www.ajtmh.org.

Acknowledgment: We thank the patient for her participation.

Authors' addresses: Lorena D.M. Gonzaga, Centro de Saude Primeiro de Maio, Maputo, Mozambique, E-mail: Imougueret@gmail.com. Tinne Gils, Tom Decroo, Bart K. M. Jacobs, and Lutgarde Lynen, Department of Clinical Sciences, Institute of Tropical Medicine, Antwerp, Belgium, E-mails: tgils@itg.be, tdecroo@itg.be, bkjacobs@ itg.be, and llynen@itg.be.

This is an open-access article distributed under the terms of the Creative Commons Attribution (CC-BY) License, which permits unrestricted use, distribution, and reproduction in any medium, provided the original author and source are credited.

\section{REFERENCES}

1. Hermans S, Caldwell J, Kaplan R, Cobelens F, Wood R, 2017. The impact of the roll-out of rapid molecular diagnostic testing for tuberculosis on empirical treatment in Cape Town, South Africa. Bull World Health Organ 95: 554-563.

2. Nguyen TNA, Anton-Le Berre V, Banuls AL, Nguyen TVA, 2019. Molecular diagnosis of drug-resistant tuberculosis; A literature review. Front Microbiol 10: 794.

3. Sanchez-Padilla E, Merker M, Beckert P, Jochims F, Dlamini T, Kahn P, Bonnet M, Niemann S, 2015. Detection of drugresistant tuberculosis by Xpert MTB/RIF in Swaziland. N Engl J Med 372: 1181-1182.

4. Makhado NA et al., 2018. Outbreak of multidrug-resistant tuberculosis in South Africa undetected by WHO-endorsed commercial tests: an observational study. Lancet Infect Dis 18: 1350-1359. 
5. Van Deun A, Aung KJ, Bola V, Lebeke R, Hossain MA, de Rijk WB, Rigouts L, Gumusboga A, Torrea G, de Jong BC, 2013. Rifampin drug resistance tests for tuberculosis: challenging the gold standard. J Clin Microbiol 51: 2633-2640.

6. Van Deun A, Decroo T, Kya Jai Maug A, Hossain MA, Gumusboga M, Mulders W, Ortuno-Gutierrez N, Lynen L, de Jong BC, Rieder HL, 2020. The perceived impact of isoniazid resistance on outcome of first-line rifampicin-throughout regimens is largely due to missed rifampicin resistance. PLoS One 15: e0233500.

7. Pauker SG, Kassirer JP, 1980. The threshold approach to clinical decision making. New Engl J Med 302: 1109-1117.

8. Van den Ende J, Mugabekazi J, Moreira J, Seryange E, Basinga P, Bisoffi Z, Menten J, Boelaert M, 2010. Effect of applying a treatment threshold in a population. An example of pulmonary tuberculosis in Rwanda. J Eval Clin Pract 16: 499-508.

9. Falzon D, Schünemann HJ, Harausz E, González-Angulo L, Lienhardt C, Jaramillo E, Weyer K, 2017. World Health Organization treatment guidelines for drug-resistant tuberculosis, 2016 update. Eur Respir J 49: 1602308.

10. Horne DJ, Kohli M, Zifodya JS, Schiller I, Dendukuri N, Tollefson D, Schumacher SG, Ochodo EA, Pai M, Steingart KR, 2019. Xpert MTB/RIF and Xpert MTB/RIF Ultra for pulmonary tuberculosis and rifampicin resistance in adults. Cochrane Database Syst Rev 6: CD009593.

11. World Health Organization, 2019. Global Tuberculosis Report 2019. Available at: https://apps.who.int/iris/bitstream/handle/ 10665/329368/9789241565714-eng.pdf?ua=1. Accessed July 29, 2020.

12. Decroo T, Henriquez-Trujillo AR, De Weggheleire A, Lynen L, 2017. Rational use of Xpert testing in patients with presumptive TB: clinicians should be encouraged to use the test-treat threshold. BMC Infect Dis 17: 674.

13. R Development Core Team, 2018. R: A Language and Environment for Statistical Computing. Vienna, Austria: R Foundation for Statistical Computing.

14. Van den Ende J, Bisoffi Z, Van Puymbroek $H$, Van der Stuyft $P$, Van Gompel A, Derese A, Lynen L, Moreira J, Janssen PA, 2007. Bridging the gap between clinical practice and diagnostic clinical epidemiology: pilot experiences with a didactic model based on a logarithmic scale. J Eval Clin Pract 13: 374-380.

15. Ministério da Saúde, 2018. República de Moçambique. Direcção Nacional de Saúde Pública Programa Nacional de Controlo da Tuberculose. Relatório das Actividades Desenvolvidas durante o ano 2018 [Report of Activitities Developped during the year 2018]. Available at: https://www.misau.gov.mz/index.php/ relatorios-anuais-pnct. Accessed December 4, 2020.

16. Boehme CC et al., 2010. Rapid molecular detection of tuberculosis and rifampin resistance. N Engl J Med 363: 1005-1015.

17. Andre E, Goeminne L, Cabibbe A, Beckert P, Kabamba Mukadi B, Mathys V, Gagneux S, Niemann S, Van Ingen J, Cambau E, 2017. Consensus numbering system for the rifampicin resistance-associated $\mathrm{rpoB}$ gene mutations in pathogenic mycobacteria. Clin Microbiol Infect 23: 167-172.

18. Ngabonziza JS et al., 2020. Reduction of diagnostic and treatment delays reduces rifampicin-resistant tuberculosis mortality in Rwanda. Int J Tuberc Lung Dis 24: 329-339.

19. Basinga P, Moreira J, Bisoffi Z, Bisig B, Van den Ende J, 2007. Why are clinicians reluctant to treat smear-negative tuberculosis? An inquiry about treatment thresholds in Rwanda. Med Decis Making 27: 53-60.

20. Boyles TH, 2017. Why do clinical trials of Xpert(R) MTB/RIF fail to show an effect on patient relevant outcomes? Int J Tuberc Lung Dis 21: 249-250.

21. Ebell MH, Locatelli I, Senn N, 2015. A novel approach to the determination of clinical decision thresholds. Evid Based Med 20: 41-47.

22. Boyles T, Locatelli I, Senn N, Ebell M, 2017. Determining clinical decision thresholds for HIV-positive patients suspected of having tuberculosis. Evid Based Med 22: 132-138.

23. Ngabonziza JCS et al., 2020. Prevalence and drivers of falsepositive rifampicin-resistant Xpert MTB/RIF results: a prospective observational study in Rwanda. Lancet Microbe 1: e74-e83.

24. Pradipta IS, Forsman LD, Bruchfeld J, Hak E, Alffenaar JW, 2018. Risk factors of multidrug-resistant tuberculosis: a global systematic review and meta-analysis. $J$ Infect 77: 469-478. 